\title{
Spatially located visual CS effects in conditioned shuttlebox avoidance in goldfish: A phototactic explanation
}

\author{
D. J. ZERBOLIO, JR. \\ University of Missouri, St. Louis, Missouri $6 \$ 121$
}

\begin{abstract}
Earlier work found that goldfish (Carassius auratus) acquire a conditioned avoidance shuttle response (CASR) differentially as a function of CS location (same, opposite, or both tank ends) when the CS is a sudden onset of illumination, and hypothesized that subjects acquire an aversion to the light. The present study finds no evidence for a conditioned aversion, but shows initial negative phototactic effects in the onset illumination situation which occurs without acquisition. Additionally, when the localized CS is a color change rather than an illumination change, the differential effects between same and both do not occur, and the very low CASR acquisition of the opposite group is strikingly similar to a no-CS group, indicating a very low-magnitude $\mathrm{CS}$ for this condition. Using the negative phototaxis explanation, the failure of subjects to acquire a CASR differentially to a light offset, which does not yield the phototactic response, is understandable.
\end{abstract}

Recently, Zerbolio and Wickstra (1976a) confirmed an earlier finding by Gallon (1974) that goldfish (Carassius auratus) acquire a conditioned avoidance shuttle response (CASR) differentially as a function of a localized light onset CS. Both Gallon (1974) and Zerbolio and Wickstra (1976a) found that, with 100 trials in a single session, CASR acquisition order from maximum to minimum according to location was same, both, opposite. To account for this order, Gallon (1974) hypothesized that subjects acquire a conditioned aversion to the $C S$ in addition to a generalized increase in activity. Thus, the conditioned aversion is compatible with and facilitates CASR acquisition performance in the same location, is incompatible with and debilitates acquisition in the opposite location, and, since the CS on both tank ends is not specifically located, both location performance is not affected. But Gallon's (1974) assumption is that the conditioned aversion is acquired. Although Zerbolio and Wickstra (1976a) supported this conclusion, Wickstra and Zerbolio (1976) found that, when subjects were run over days instead of a single day, CASR performance order from maximum to minimum was both, same, opposite, which does not agree with the conditioned aversion hypothesis but does follow a CS magnitude dimension (Kish, 1955). Additionally, Zerbolio and Wickstra (1976b) found that, with ITI illumination held constant and a localized color change as the CS, both and same locations were equivalent in the 1-day paradigm, with opposite much lower, and with training over days, both, same, and opposite locations were equivalent. To account for the discrepancies between the illumination change CS and the color change CS CASR acquisition orders, Zerbolio and Wickstra (1976b) hypothesized that the illumination change produces a negative phototaxis, whereas the color change does not. Thus, the illumination change affects performance (debilitates the opposite and both conditions and facilitates the same performance) from the first trial and is not acquired. With illumination during the ITI, and a localized color change CS, the order of CASR acquisition would be expected to follow the magnitude of CS change, which is comparable for the same and both locations but markedly less for the opposite location. These effects, especially the phototaxis in the illumination change paradigm, would be expected to be operating at the onset of training and would not require any sort of acquisition, as does an hypothesized conditioned aversion operator. The present study partially replicates the 1-day illumination change and color change portions of Wickstra and Zerbolio (1976) and Zerbolio and Wickstra (1976b) but includes an additional measure, trial-by-trial latency. It is expected that the latency measure, although providing information similiar to the avoidance information, will be more sensitive to the phototaxis, especially in the early trial blocks, than the CASR acquisition rate.

\section{METHOD}

\section{Subjects}

Eighty goldfish, from Ozark Fisheries, housed upon receipt in 30-gal aquaria, were used. Forty-eight hours prior to use, the subjects were transferred to 10-gal holding aquaria in the experimental chamber, where temperature $\left(21.1^{\circ} \mathrm{C}\right)$ and $\mathrm{pH}(7 \pm .1)$ were held constant. All subjects were housed in well aerated and filtered tanks, and were fed daily throughout.

\footnotetext{
Apparatus

Subjects were run in two identical $29.2 \times 11.4 \times 11.4 \mathrm{~cm}$ deep shuttle tanks, with a $6.35-\mathrm{cm}$ hurdle and $2.5-\mathrm{cm}$ water clearance.
} 


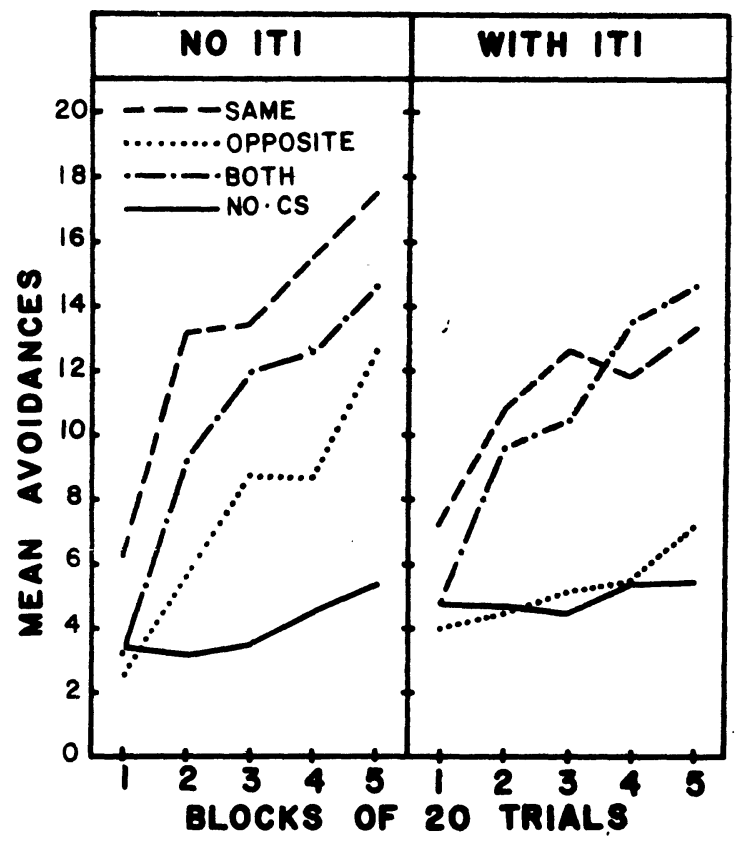

Figure 1. The mean avoidances for the same-, opposite-, and both-end CS location groups and the no-CS groups, both with ITI illumination and with no-ITI illumination by 20 trial blocks.

Light-diffusing plates were affixed to the tank ends and the US was delivered via $28 \times 10.2 \mathrm{~cm} 22-\mathrm{g}$ stainless steel plates affixed to the interior sides. The US was generated by individually isolated, metered, and monitored variable transformers set at $7.5 \mathrm{~V}$ ac. Activity was monitored by twe photocell units set across the ends of the $7.6-\mathrm{cm}$ hurdle top. The CS or ITI lights were individually programmable 7-W ac lamps, placed near the tank ends. Each end had both a blue and green lamp, generating light well within the visual spectrum of goldfish (Yager, 1968).

\section{Procedure}

A factorial arrangement of 2 ITI conditions (no ITI illumination and with green ITI illumination) by 3 CS locations (same, opposite, and both tank ends) constituting 6 groups of 10 subjects each were run. An additional two groups of 10 subjects each, with no CS but under the ITI conditions above, were also run. Each subject had 100 VI 60 -sec trials in a single session, immediately following a 30-min presession period during which shuttle rate activity was measured. A trial started with a 10 -sec CS, followed by a maximum of five $200-\mathrm{msec}$-on/300-msec-off $7.5-\mathrm{V}$ ac shocks, for a total 12.2-sec trial period. The CS was a $7-\mathrm{W}$ blue $110 \mathrm{ac}$ lamp, onset in the no-ITI illumination condition and a green to blue color change in the with- (green) ITI illumination condition; the no-CS groups had no stimulus change to indicate trial onset.

On any trial, the initial shuttle terminated all stimulation (CS and/or US) immediately. If the initial shuttle occurred in the 10-sec CS-US period, it was recorded as an avoidance; in the 2.2-sec US period, it was recorded as an escape. Additionally, total shuttles during the ITI and trial-by-trial latency to the nearest $.2 \mathrm{sec}$ were recorded.

\section{RESULTS AND DISCUSSION}

Figure 1 shows the mean avoidance for the same, opposite, and both CS locations and for the no-CS groups. The no-CS groups showed no differences in avoidance rate $[F(1,18)=.603]$ and no Training effect $[F(4,72)=1.997]$. For the same, opposite, and both groups, significant effects for CS Location
$[F(2,54)=8.296, p<.01]$, Training $[F(4.216)=$ 178.26, $\mathrm{p}<.01]$, ITI Illumination by Training interaction $[F(4.216)=11.46, \quad \mathrm{p}<.01]$, and $C S$ Location by Training interaction $[\mathrm{F}(8,216)=6.20$, $p<.01]$ were found. A partial of the CS Location by Training interaction shows the significant portion to be in the with-ITI illumination condition $[F(4,216)=$ $6.89, \mathrm{p}<.01]$, while in the no-ITI illumination condition, there is no significant effect $[F(4,216)=$ $2.32, \mathrm{p}>.05]$. This latter finding indicates that, in the no-ITI condition, the same, opposite, and both CS location differences are present at the onset of training and are not acquired. A similar analysis for the latency measure was performed and mean latencies appear in Figure 2. As before, the no-CS groups did not show ITI illumination differences $[F(1.18)=.261]$ or Training effects $[F(4.72)=$ 1.36]. For the same, opposite, and both CS Location by ITI Illumination analysis, significant effects for CS Location $[\mathrm{F}(2,54)=8.820, \mathrm{p}<.01]$, Training effects $[F(4,216)=61.998, p<.01]$, ITI by Training interaction $[F(4,226)=3.023, p<.05]$, and $C S$ Location by Training interaction $[F(8,216)=$ 4.329, $\mathrm{p}<.01$ ] were found. Again, a partial of the CS Location by Training interaction found no effect in the no-ITI illumination condition $[F(4,216)=$ 1.42] and significant effects in the with-ITI illumination condition $[F(4,216=3.37, p<.05]$. Plotted in the first trial block is a .05 HSD interval (Winer, 1971) indicating no initial trial block differences in the with-ITI illumination condition; there are significant differences between the same CS location and the both and opposite CS locations. This analysis indicates that, instead of being acquired, the same,

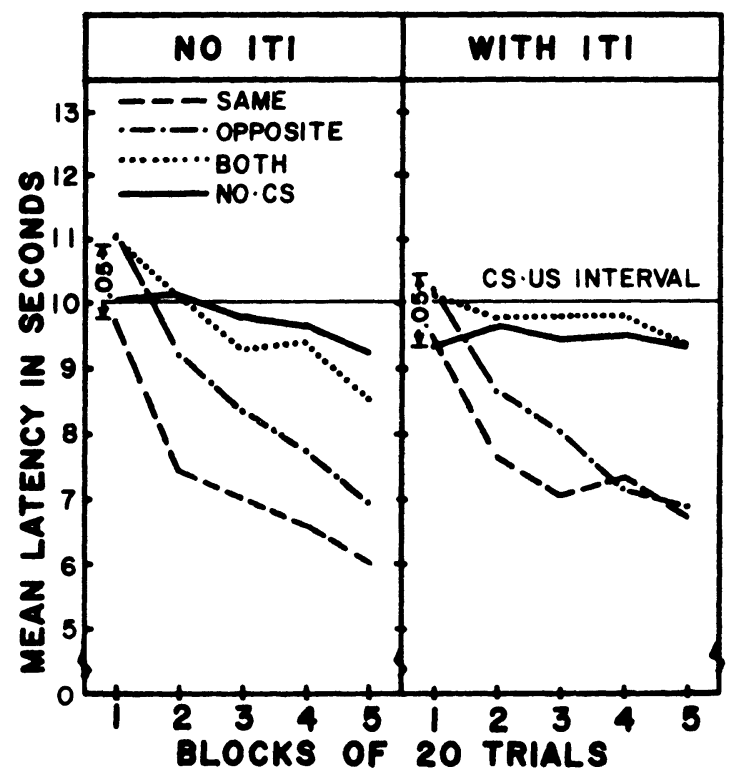

Figure 2. The mean response latencies in seconds for the same-, opposite-, and both-end CS location groups and the no-CS groups, both with ITI illumination and with no-ITI illumination by 20 trial blocks. A $5 \%$ difference interval for the first 20 trials and the CS.US interval is indicated. 


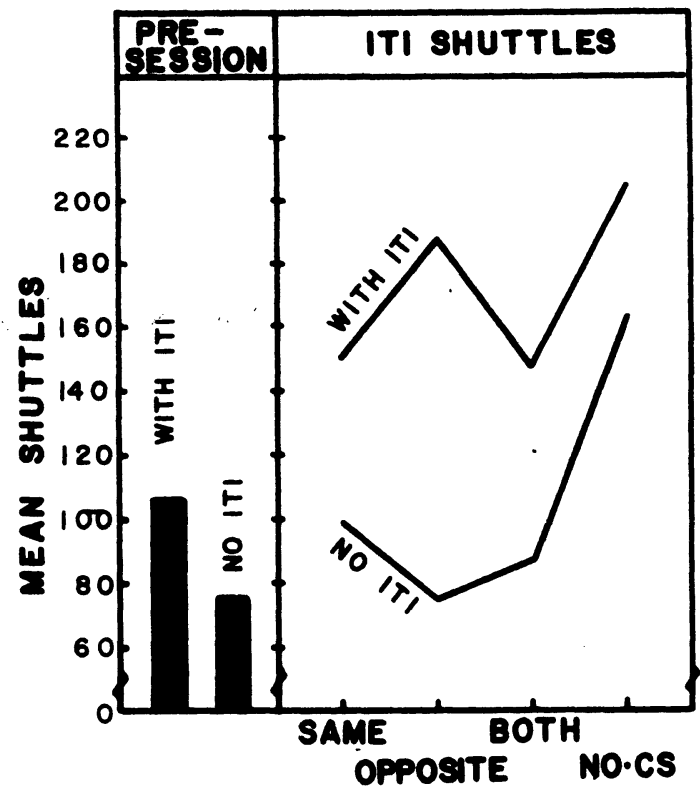

Figure 3. The mean shuttle rate for the 30-min presession period with ITI and with no-ITI illumination and the mean shuttle rate for CS location and no CS by ITI illumination condition are shown.

opposite, and both CS location differences are present in the first trial block under the no-ITI condition, but not present with the green ITI illumination. A further analysis on the presession and ITI shuttle rate measures was performed and mean shuttle rates appear in Figure 3. For presession rates, having the green illumination produced higher shuttle rates than no illumination $[F(3,72)=7.590, p<.01]$. For ITI shuttle rates, both CS Location (or no CS) $[\mathrm{F}(3,72)=3.669, \mathrm{p}<.05]$ and ITI Illumination $[F(1,72)=6.826, p<.01]$ reached significance. What is striking about these data is that the no-CS conditions and the with-ITI illumination opposite conditions all have very high activity rates. This finding strongly supports Zerbolio and Wickstra's (1976b) suggestion that the opposite color change over days acquisition effect is a huge increase in shuttling activity.

In sum, then, the following data clearly show that, with no-ITI illumination, a localized CS (light onset) produces immediate and not differentially acquired CS location effects on CASR acquisition. A negative phototaxis explanation is consistent with this interpretation. Additionally, if one assumes the negative phototaxis, the failure to find differential conditioning to a localized area of darkness (Gallon, 1974; Zerbolio \& Wickstra, 1976a) also can be retrodicted, since the sudden decrease in illumination does not produce a negative phototactic response. As a result, the decrease in illumination by CS location conditions would be expected to produce roughly equivalent CASR acquisition rates, as found by Zerbolio and Wickstra (1976a), although not by Gallon (1974). The remaining question is why the CS opposite with-ITI illumination condition shows such a low CASR acquisition rate. The present finding suggests that, with ITI illumination, a color change on the tank end opposite the subject has a very low CS magnitude, much lower than CS opposite with no ITI. The similarity in all measures, CASR acquisition, latency, and ITI shuttle rate for the with-ITI CS opposite group and the no-CS groups, supports this interpretation. One question remaining is, will the no-CS conditions, if trained over days, show an increase in shuttle rate similar to the behavior of the CS opposite color change group found by Zerbolio and Wickstra (1976b); if so, will this be associated with a concomitant increase in avoidance performance? If the shuttle rate increase for the no-CS groups occurs without an increase in avoidance rate, it would imply that the CS opposite color change groups can, if trained over days, learn a CASR even under very minimum CS conditions. This is, however, a matter for further investigation.

\section{REFERENCES}

Gallon, R. L. Spatial location of a visual signal and shuttle box avoidance acquisition by goldfish (Carassius auratus). Journal of Comparative and Physiological Psychology, 1974, 86, 316-321.

KISH, G. B. Avoidance learning to the onset and cessation of conditioned stimulus energy. Journal of Experimental Psychology, 1955, 50, 31-38.

Wickstra, L. L., \& Zerbolio, D. J., JR. Spatially located visual CS effects in conditioned avoidance shuttle response (CASR) acquisition in goldfish (Carassius auratus): Training over days. Bulletin of the Psychonomic Society, 1976, 8, 124-126.

WINER, B. J. Statistical principles in experimental design (2nd ed.). New York: McGraw-Hill, 1971.

YAGER, D. Behavioral analysis of color sensitivities in goldfish. In D. Ingle (Ed.), The central nervous system and fish behavior. Chicago: University of Chicago Press, 1968.

Zerbolio, D. J., JR., \& WickstrA, L. L. Spatially located visual CS effects on conditioned shuttlebox avoidance in goldfish (Carassius auratus): Further analysis. Bulletin of the Psychonomic Society, 1976, 7, 503-505. (a)

Zerbolio, D. J., JR., \& WiCkstrA, L. L. Spatially located visual CS effects in conditioned avoidance shuttle acquisition in goldfish: Conditioned aversion or phototaxis. Bulletin of the Psychonomic Society, 1976, 8, 156-158. (b)

(Received for publication July 11, 1976.) 